\title{
Short Synthesis of the C1-C14 Stretch of Discodermolide from Building Blocks Prepared by Asymmetric Catalysis
}

\author{
Huanyan Cao and Kathlyn A. Parker* \\ Department of Chemistry, State University of New York at Stony Brook \\ Stony Brook, New York 11794-3400 \\ kparker@notes.cc.sunysb.edu
}

Supporting Information

\section{Table of Contents}

\section{Part 1. Analytical Data and Discussion}

General Information

Experimental Procedures for Compounds 2 and 10-19 .............................2

Assignment of Stereochemistry to Alcohol 15 and its Epimer by Application

of the Rychnovsky Method to the Corresponding Diols.

Assignment of Stereochemistry to Alcohol 11 and its Epimer by Application

of the "Broadened" Version of the Advanced Mosher Ester Method.............. 12

${ }^{1} \mathrm{H}$ and ${ }^{13} \mathrm{C}$ NMR Spectra for Compounds 2 and 10-19

${ }^{1} \mathrm{H}$ and ${ }^{13} \mathrm{C}$ NMR Spectra for Diols and Acetonides Prepared

for the Rychnovsky Analysis

${ }^{1} \mathrm{H}$ NMR Spectra for Derivatives Prepared for the Mosher Ester Analysis

Part 2. Index of Compounds

\begin{tabular}{|c|c|c|}
\hline Structure & Experimental & NMR Spectra \\
\hline 14 & 2 & $17-18$ \\
\hline 17 & 3 & $19-20$ \\
\hline 15 and its (4R)-epimer & 3 & $21-24$ \\
\hline 16 & 4 & 25 \\
\hline 12 & 4 & $26-27$ \\
\hline 13 & 5 & $28-29$ \\
\hline 11 and its (7R)-epimer & 5 & $30-33$ \\
\hline MOM-ether of 11 & 6 & $34-35$ \\
\hline 18 & 7 & $36-37$ \\
\hline 19 & 8 & $38-39$ \\
\hline 10 & 8 & $40-41$ \\
\hline 2 & 9 & $42-43$ \\
\hline
\end{tabular}




\section{General Information}

Unless noted otherwise, all oxygen and moisture-sensitive reactions were executed in oven-dried glassware sealed under a positive pressure of dry argon and moisture sensitive solutions and anhydrous solvents were transferred via standard syringe and cannula techniques. Unless stated otherwise, all commercial reagents, were used as received. All solvents were dried under argon atmosphere: THF and diethyl ether were distilled over Na-benzophenone; $\mathrm{CH}_{2} \mathrm{Cl}_{2}$, were distilled from $\mathrm{CaH}_{2}$. The extracts were dried over $\mathrm{Na}_{2} \mathrm{SO}_{4}$ unless otherwise noted. Flash chromatography was performed using Scientific Flash silica gel (32-63 mM); analytical TLC was performed using 0.25 mm EM silica gel 60 F254 plates that were visualized by irradiation $(254 \mathrm{~nm})$ or by staining $10 \%$ PMA. IR spectra were recorded using a Galaxy Series FTIR 3000 infrared spectrophotometer. NMR spectra were obtained using Gemini-300MHz (Varian), Inova-400 MHz (Varian) Inova-500 MHz (Varian) and Inova-600 MHz (Varian) instruments.

\section{Experimental Procedures}

Aldehyde 14. To a stirred mixture of alcohol 9a $(220 \mathrm{mg}, 0.64 \mathrm{mmol})$ and powdered molecular sieves 4A (600 mg) in $10 \mathrm{~mL}$ of methylene chloride were added NMO (226 $\mathrm{mg}, 1.93 \mathrm{mmol})$ followed by TPAP $(15.8 \mathrm{mg}, 44.9 \mu \mathrm{mol})$. After stirred at rt. for $30 \mathrm{~min}$, the mixture was filtered through a silica gel column (HE: EA = 10:1) gave aldehyde 14 (215 mg, 98\%) as a colorless oil. ${ }^{1} \mathrm{H}$ NMR $\left(300 \mathrm{MHz}, \mathrm{CDCl}_{3}\right) \delta 9.69$ (d, J = $\left.1.9 \mathrm{~Hz}, 1 \mathrm{H}\right)$, $5.39(\mathrm{dd}, \mathrm{J}=15.4,6.0 \mathrm{~Hz}, 1 \mathrm{H}), 5.16(\mathrm{ddd}, \mathrm{J}=15.4,8.0,1.1 \mathrm{~Hz}), 3.75$ (dd, J = 6.9, $3.3 \mathrm{~Hz}$, $1 \mathrm{H}), 2.51(\mathrm{~m}, 1 \mathrm{H}), 2.37(\mathrm{~m}, 1 \mathrm{H}), 1.87(\mathrm{~m}, 1 \mathrm{H}), 1.73-1.60(\mathrm{~m}, 4 \mathrm{H}), 1.32-1.02(\mathrm{~m}, 6 \mathrm{H})$, $1.11(\mathrm{~d}, \mathrm{~J}=6.9 \mathrm{~Hz}, 3 \mathrm{H}), 0.99$ (d, J = 6.9 Hz, 3H), 0.88 (s, 9H), 0.08 (s, 3H), 0.06 (s, 3H);

${ }^{13} \mathrm{C} \mathrm{NMR}\left(75 \mathrm{MHz}, \mathrm{CDCl}_{3}\right) \delta 204.8,138.5,129.3,78.8,50.7,41.9,41.0,33.0,32.9,26.5$, 26.3, 26.2, 18.5, 17.6, 11.4, -3.8, -4.1; IR (neat) $v_{\max } 2956,2928,2854,1724,1255,1079$, 1038, 852; HRMS (FAB) calcd for $\mathrm{C}_{20} \mathrm{H}_{38} \mathrm{O}_{2} \mathrm{Si}(\mathrm{M})^{+}$338.2641, found 338.2637. 
Aldehyde 17. Aldehyde 17 was prepared in $88 \%$ yield by a procedure similar to that above from alcohol 9b. ${ }^{1} \mathrm{H}$ NMR $\left(400 \mathrm{MHz}, \mathrm{CDCl}_{3}\right) \delta 9.68(\mathrm{~d}, \mathrm{~J}=1.8 \mathrm{~Hz}, 1 \mathrm{H}), 5.40$ (dd, $\mathrm{J}=15.6,6.6 \mathrm{~Hz}, 1 \mathrm{H}), 5.16(\mathrm{ddd}, \mathrm{J}=15.6,8.2,1.3 \mathrm{~Hz}, 1 \mathrm{H}), 4.66(\mathrm{~d}, \mathrm{~J}=7.1 \mathrm{~Hz}, 1 \mathrm{H}), 4.64$ (d, J = 7.0 Hz, 1H), 3.63 (dd, J = 7.0, $4.4 \mathrm{~Hz}, 1 \mathrm{H}), 3.35$ (s, 3H), $2.64(\mathrm{~m}, 1 \mathrm{H}), 2.40$ (m, 1H), $1.86(\mathrm{~m}, 1 \mathrm{H}), 1.72-1.55(\mathrm{~m}, 4 \mathrm{H}), 1.32-1.02(\mathrm{~m}, 6 \mathrm{H}), 1.11(\mathrm{~d}, \mathrm{~J}=7.1 \mathrm{~Hz}, 3 \mathrm{H}), 1.03$ $(\mathrm{d}, \mathrm{J}=6.8 \mathrm{~Hz}, 3 \mathrm{H}) ;{ }^{13} \mathrm{C} \mathrm{NMR}\left(75 \mathrm{MHz}, \mathrm{CDCl}_{3}\right) \delta 203.9,138.5,129.3,97.7,84.2,56.3$, 49.3, 40.9, 39.9, 33.01, 32.98, 26.4, 26.3, 16.8, 11.0; IR (neat) $v_{\max } 2926,2853,1724$, 1147, 1099, 1033; HRMS (FAB) calcd for $\mathrm{C}_{16} \mathrm{H}_{29} \mathrm{O}_{3}\left[(\mathrm{M}+\mathrm{H})^{+}\right]$269.2117, found 269.2124.

Alcohol 15 and its $4 \mathbf{R}$ Epimer. To the solution of (-)- Bmethoxydiisopinocampheylborane $(215 \mathrm{mg}, 0.680 \mathrm{mmol})$ in $5 \mathrm{~mL}$ of ether was added allyl magnesium bromide $(1.0 \mathrm{M}$ in ether, $649 \mu \mathrm{L}, 0.649 \mathrm{mmol})$ dropwise at room temperature. After $1 \mathrm{~h}$ at room temperature, the reaction mixture was cooled to $-78{ }^{\circ} \mathrm{C}$. Aldehyde 14 (210 mg, $0.618 \mathrm{mmol})$ in $2 \mathrm{~mL}$ of ether was added dropwise. After being stirred for $5 \mathrm{~h}$ at $-78^{\circ} \mathrm{C}$ and $5 \mathrm{~h}$ at $-40^{\circ} \mathrm{C}$, the reaction was added $1 \mathrm{~mL}$ of $3 \mathrm{~N} \mathrm{NaOH}$ and $1 \mathrm{~mL}$ of $30 \% \mathrm{H}_{2} \mathrm{O}_{2}$ and warmed to room temperature. The mixture was refluxed $2 \mathrm{~h}$ and then poured into brine $(10 \mathrm{~mL})$. The aqueous phase was extracted with ether and the organic phase was combined, dried $\left(\mathrm{MgSO}_{4}\right)$, and concentrated. Chromatography (HE: $\left.\mathrm{CH}_{2} \mathrm{Cl}_{2}=1: 1\right)$ first gave alcohol $15(168 \mathrm{mg}, 71 \%)$ as a colorless oil and then its $4 \mathrm{R}$ epimer (40.1 $\mathrm{mg}, 17 \%)$ as a colorless oil.

For alcohol 15: ${ }^{1} \mathrm{H}$ NMR $\left(300 \mathrm{MHz}, \mathrm{CDCl}_{3}\right) \delta 5.90(\mathrm{~m}, 1 \mathrm{H}), 5.30(\mathrm{~m}, 2 \mathrm{H}), 5.11(\mathrm{~d}, \mathrm{~J}=$ $1.5 \mathrm{~Hz}, 1 \mathrm{H}), 5.07(\mathrm{~m}, 1 \mathrm{H}), 3.64$ (tt, J = 8.5, $2.7 \mathrm{~Hz}, 1 \mathrm{H}), 3.53(\mathrm{dd}, \mathrm{J}=6.0,4.6 \mathrm{~Hz}, 1 \mathrm{H})$, $2.93(\mathrm{~d}, \mathrm{~J}=2.5 \mathrm{~Hz}, 1 \mathrm{H}), 2.42-2.28(\mathrm{~m}, 2 \mathrm{H}), 2.14-2.02(\mathrm{~m}, 1 \mathrm{H}), 1.98-1.60(\mathrm{~m}, 7 \mathrm{H}), 1.38-$ 1.01, (m, 5H), 0.97 (d, J = 6.9 Hz, 3H), 0.92 (s, 9H), 0.85 (d, J = 6.9 Hz, 3H), 0.09 (s, 3H), $0.08(\mathrm{~s}, 3 \mathrm{H}) .{ }^{13} \mathrm{C} \mathrm{NMR}\left(75 \mathrm{MHz}, \mathrm{CDCl}_{3}\right) \delta 137.0,135.8,131.0,117.3,80.9,72.8,42.8$, $42.1,41.0,39.0,33.4,33.3,26.5,26.38,26.35,18.5,17.1,15.3,-3.7,-3.8$; IR (neat) $v_{\max }$ 3500 (broad), 2926, 2854, 1462, 1449, 1254, 1003, 974, 835; HRMS (FAB) calcd for $\mathrm{C}_{23} \mathrm{H}_{45} \mathrm{O}_{2} \mathrm{Si}\left[(\mathrm{M}+\mathrm{H})^{+}\right]$381.3189, found 381.3160 . 
For its $4 \mathrm{R}$ epimer ${ }^{1} \mathrm{H}$ NMR (300 MHz, $\left.\mathrm{CDCl}_{3}\right) \delta 5.76(\mathrm{~m}, 1 \mathrm{H}), 5.31(\mathrm{~m}, 2 \mathrm{H}), 5.12-4.98$ (m, 2H), 4.20 (t, J = 6.7 Hz, 1H), 3.52 (dd, J = 7.7, $2.2 \mathrm{~Hz}, 1 \mathrm{H}), 3.45$ (s, 1H), 2.47 (m, $1 \mathrm{H}), 2.28(\mathrm{~m}, 1 \mathrm{H}), 2.03(\mathrm{~m}, 1 \mathrm{H}), 1.98-1.60(\mathrm{~m}, 7 \mathrm{H}), 1.38-1.01$, (m, 5H), $1.01(\mathrm{~d}, \mathrm{~J}=6.9$ $\mathrm{Hz}, 3 \mathrm{H}), 0.98$ (d, J = $6.9 \mathrm{~Hz}, 3 \mathrm{H}), 0.92(\mathrm{~s}, 9 \mathrm{H}), 0.13$ (s, 3H), 0.11 (s, 3H). ${ }^{13} \mathrm{C}$ NMR (75 $\left.\mathrm{MHz}, \mathrm{CDCl}_{3}\right) \delta 136.8,135.9,130.1,116.9,83.8,70.5,42.0,41.1,39.6,37.2,33.38$, 33.32, 26.5, 26.4, 18.6, 12.3, -3.3, -3.6; IR (neat) $v_{\max } 3505$ (broad), 2926, 2854, 1462, 1449, 1255, 1084, 1019, 1003; HRMS (FAB) calcd for $\mathrm{C}_{23} \mathrm{H}_{45} \mathrm{O}_{2} \mathrm{Si}\left[(\mathrm{M}+\mathrm{H})^{+}\right]$381.3189, found 381.3178 .

Aldehyde 16. To alcohol $15(191 \mathrm{mg}, 0.500 \mathrm{mmol})$ in $15 \mathrm{~mL}$ of $\mathrm{CH}_{2} \mathrm{Cl}_{2}$ at $-78{ }^{\circ} \mathrm{C}, \mathrm{O}_{3}$ was flushed in until the solution turned into blue. After the solution being flushed with argon for $10 \mathrm{~min}$ at $-78{ }^{\circ} \mathrm{C}, \mathrm{Ph}_{3} \mathrm{P}(327 \mathrm{mg}, 1.25 \mathrm{mmol})$ was added. After $1 \mathrm{~h}$ at $-78{ }^{\circ} \mathrm{C}$ and $6 \mathrm{~h}$ at room temperature, the mixture was concentrated. Chromatography (HE: EA = 5: 1) gave compound 16 as a colorless oil (125 mg, 91\%). ${ }^{1} \mathrm{H}$ NMR (300 MHz, $\left.\mathrm{CDCl}_{3}\right) \delta$ $9.82(\mathrm{~m}, 1 \mathrm{H}), 5.48(\mathrm{~d}, \mathrm{~J}=10.7 \mathrm{~Hz}, 0.5 \mathrm{H}), 5.22(\mathrm{~s}, 0.5 \mathrm{H}), 4.83(\mathrm{~d}, \mathrm{~J}=10.4,0.5 \mathrm{H}), 4.38(\mathrm{~m}$, $0.5 \mathrm{H}), 4.08(\mathrm{~m}, 0.5 \mathrm{H}), 3.74(\mathrm{~s}, 0.5 \mathrm{H}), 3.66(\mathrm{t}, \mathrm{J}=2.5 \mathrm{~Hz}, 0.5 \mathrm{H}), 2.65-2.40(\mathrm{~m}, 2 \mathrm{H})$, 2.10-1.62 (m, 2H), 1.03-0.79 (m, 15H), 0.14 (s, 1.5H), 0.10 (s, 1.5H), 0.06 (s, 1.5H), 0.04 (s, 1.5H); IR (neat) $v_{\max } 3450$ (broad), 2962, 2930, 1724, 1708, 1172,1154, 1109, 1095; HRMS (FAB) calcd for $\mathrm{C}_{15} \mathrm{H}_{29} \mathrm{O}_{4} \mathrm{Si}\left[(\mathrm{M}-\mathrm{H})^{+}\right]$301.1835, found 301.1828 .

Aldehyde 12. To a solution of aldehyde $16(120 \mathrm{mg}, 0.438 \mathrm{mmol})$ in $1 \mathrm{~mL}$ of $\mathrm{CH}_{2} \mathrm{Cl}_{2}$ was added imidazole $(44.7 \mathrm{mg}, 0.657 \mathrm{mmol})$, DMAP (13.4mg, $0.11 \mathrm{mmol})$, TBDMSCl (198 mg, $1.31 \mathrm{mmol})$ at $0{ }^{\circ} \mathrm{C}$. The mixture was stirred for $12 \mathrm{~h}$ and then directly loaded on silica gel column. Chromatography (HE: EA = 10: 1) gave compound 12 as a colorless oil (128 mg, 70\%). ${ }^{1} \mathrm{H}$ NMR $\left(300 \mathrm{MHz}, \mathrm{CDCl}_{3}\right) \delta 9.82(\mathrm{t}, \mathrm{J}=2.7 \mathrm{~Hz}, 1 \mathrm{H}), 5.21$ (d, J = 3.0 Hz, 1H), 4.05 (dt, J = 10.2, 6.3 Hz, 1H), $3.66(\mathrm{t}, \mathrm{J}=2.7 \mathrm{~Hz}, 1 \mathrm{H}), 2.49(\mathrm{~m}, 2 \mathrm{H})$, 1.88-1.77 (m, 1H), 1.76-1.64 (m, 1H), $0.94(\mathrm{~d}, \mathrm{~J}=7.2 \mathrm{~Hz}, 3 \mathrm{H}), 0.92$ (s, 9H), $0.88(\mathrm{~s}, 9 \mathrm{H})$, $0.80(\mathrm{~d}, \mathrm{~J}=7.0 \mathrm{~Hz}, 3 \mathrm{H}), 0.07$ (s, 3H), $0.06(\mathrm{~s}, 6 \mathrm{H}), 0.05$ (s, 3H); ${ }^{13} \mathrm{C} \mathrm{NMR}(75 \mathrm{MHz}$, $\left.\mathrm{CDCl}_{3}\right) \delta 202.5,94.6,76.6,72.2,46.9,43.0,34.8,26.1,26.0,18.3,13.8,9.60,-3.9,-4.3,-$ 4.7, -5.1; IR (neat) $v_{\max } 2955,2928,1731,1462,1174,1120,1049,836$; HRMS (FAB) calcd for $\mathrm{C}_{21} \mathrm{H}_{43} \mathrm{O}_{4} \mathrm{Si}_{2}\left[(\mathrm{M}-\mathrm{H})^{+}\right]$415.2700, found 415.2679. 
Alkyne 13. To a cooled $\left(-78{ }^{\circ} \mathrm{C}\right)$ solution of dimethyl 1-diazophosphonoacetone (OhiraBestmann's reagent, $461 \mathrm{mg}, 2.40 \mathrm{mmol}$ ) in $10 \mathrm{~mL}$ of THF was added sodium methoxide (0.5 $\mathrm{M}$ in THF, $4.80 \mathrm{~mL}, 2.40 \mathrm{mmol}$ ) dropwise via cannula. After stirring for $15 \mathrm{~min}$, a solution of aldehyde $17(160 \mathrm{mg}, 0.60 \mathrm{mmol})$ in $3 \mathrm{~mL}$ of THF was added via cannula. The reaction mixture was allowed to slowly warm to room temperature over $1 \mathrm{~h}$ and quenched with saturated aqueous ammonium chloride solution $(3 \mathrm{~mL})$. The mixture was then diluted with water $(15 \mathrm{~mL})$ and then extracted with ether $(3 \times 15 \mathrm{~mL})$. The combined extracts were washed with brine, dried over sodium sulfate and concentrated. Chromatographed (HE: EA = 10: 1) afforded acetylene $13(136 \mathrm{mg}, 85 \%)$ as a colorless oil. ${ }^{1} \mathrm{H}$ NMR (400 MHz, $\left.\mathrm{CDCl}_{3}\right) \delta 5.46(\mathrm{dd}, \mathrm{J}=15.4,6.6 \mathrm{~Hz}, 1 \mathrm{H}), 5.24(\mathrm{ddd}, \mathrm{J}=15.4$, 8.2, 1.2 Hz, 1H), 4.74 (d, J = 7.0 Hz, 1H), 4.70 (d, J = 7.0 Hz, 1H), 3.42 (s, 3H), 3.14, (dd, $\mathrm{J}=7.9,3.5 \mathrm{~Hz}, 1 \mathrm{H}), 2.76(\mathrm{~m}, 1 \mathrm{H}), 2.49(\mathrm{~m}, 1 \mathrm{H}), 2.07$ (d, J = 2.6 Hz, 1H), 1.96-1.84 (m, 1H), 1.72-1.58 (m, 5H), $1.23(\mathrm{~d}, \mathrm{~J}=6.7 \mathrm{~Hz}, 3 \mathrm{H}), 1.18-1.07(\mathrm{~m}, 5 \mathrm{H}), 1.04(\mathrm{~d}, \mathrm{~J}=6.8 \mathrm{~Hz}$, $3 \mathrm{H}) ;{ }^{13} \mathrm{C} \mathrm{NMR}\left(100 \mathrm{MHz}, \mathrm{CDCl}_{3}\right) \delta 137.2,130.1,98.5,86.3,85.9,70.1,70.0,56.5,41.0$, 40.9, 33.2, 33.1, 29.9, 26.4, 26.3, 18.6, 17.2; IR (neat) $v_{\max } 2926,2851,1149,1096,1035$; HRMS (FAB) calcd for $\mathrm{C}_{17} \mathrm{H}_{27} \mathrm{O}_{2}\left[(\mathrm{M}-\mathrm{H})^{+}\right]$263.2011, found 263.2019.

Alcohol 11 and its 7R Epimer. To a cold solution $\left(-50^{\circ} \mathrm{C}\right)$ of alkyne $13(125 \mathrm{mg}, 0.470$ mmol) was added BuLi (2.5 $\mathrm{M}$ in hexanes, $188 \mu \mathrm{L}, 0.470 \mathrm{mmol})$ dropwise. The temperature was increased to $-40{ }^{\circ} \mathrm{C}$ over $1 \mathrm{~h}$ and then cooled to $-78{ }^{\circ} \mathrm{C}$. Aldehyde 12 (49.0 mg, $0.118 \mathrm{mmol}$ ) was added dropwise in $1 \mathrm{~mL}$ of THF. The temperature was increased to $-40^{\circ} \mathrm{C}$ and stirred for $5 \mathrm{~h}$. Water $(1 \mathrm{~mL})$ was added dropwise at $-40{ }^{\circ} \mathrm{C}$ and the mixture was warmed to room temperature. The aqueous phase was extracted with ether and organic phases were combined, dried $\left(\mathrm{Na}_{2} \mathrm{SO}_{4}\right)$. Chromatography (HE: EA = 10: 1) first recovered excess alkyne 13, then gave compound 11 (51.4 mg, 64\%) and its 7R isomer (14.7 mg, 18\%).

For compound 11: ${ }^{1} \mathrm{H}$ NMR $\left(600 \mathrm{MHz} \mathrm{CDCl}_{3}\right) \delta 5.44(\mathrm{dd}, \mathrm{J}=15.5,6.4 \mathrm{~Hz}, 1 \mathrm{H}), 5.31$ (dd, J = 15.5, 7.9 Hz, 1H), $5.21(\mathrm{~d}, \mathrm{~J}=2.3 \mathrm{~Hz}, 1 \mathrm{H}), 4.72$ (d, J = 7.0 Hz, 1H), 4.67 (d, J = $6.8 \mathrm{~Hz}, 1 \mathrm{H}), 4.63(\mathrm{t}, \mathrm{J}=6.8 \mathrm{~Hz}, 1 \mathrm{H}), 4.05(\mathrm{td}, \mathrm{J}=10.0,2.6 \mathrm{~Hz}, 1 \mathrm{H}), 3.64(\mathrm{~s}, 1 \mathrm{H}), 3.52(\mathrm{~d}$, 
$\mathrm{J}=9.6 \mathrm{~Hz}, 1 \mathrm{H}), 3.40(\mathrm{~s}, 3 \mathrm{H}), 3.17(\mathrm{dd}, \mathrm{J}=7.0,4.4 \mathrm{~Hz}, 1 \mathrm{H}), 2.79(\mathrm{~m}, 1 \mathrm{H}), 2.45(\mathrm{~m}, 1 \mathrm{H})$, 1.98-1.60 (m, 5H), $1.20(\mathrm{~d}, \mathrm{~J}=7.0 \mathrm{~Hz}, 3 \mathrm{H}), 1.18-1.07(\mathrm{~m}, 5 \mathrm{H}), 1.03(\mathrm{~d}, \mathrm{~J}=6.7 \mathrm{~Hz}, 3 \mathrm{H})$, 0.93 (d, J = 7.0 Hz, 3H), 0.90 (s, 9H), 0.88 (s, 9H), 0.78 (d, J = 6.8 Hz, 3H), 0.11 (s, 3H), 0.10 (s, 3H), 0.05 (s, 3H), 0.03 (s, 3H). ${ }^{13} \mathrm{C}$ NMR $\left(75 \mathrm{MHz}, \mathrm{CDCl}_{3}\right) \delta 136.9,130.5,98.4$, 94.4, 86.9, 85.9, 83.1, 76.9, 74.3, 61.0, 56.4, 43.2, 41.0, 40.6, 39.8, 34.6, 33.4, 33.3, 30.2, $26.5,26.4,26.1,26.0,18.5,18.4,18.3,16.8,14.0,9.7,-4.0,-4.2,-4.7,-5.2$; IR (neat) $v_{\max }$ 3580 (broad), 2955, 2856, 1105, 1049, 1035; HRMS (FAB) calcd for $\mathrm{C}_{38} \mathrm{H}_{71} \mathrm{O}_{6} \mathrm{Si}_{2}$ [(M$\left.\mathrm{H})^{+}\right] 679.4789$, found 679.4756 .

For its 7R epimer: ${ }^{1} \mathrm{H}$ NMR $\left(600 \mathrm{MHz}, \mathrm{CDCl}_{3}\right) \delta 5.43(\mathrm{dd}, \mathrm{J}=15.5,6.6 \mathrm{~Hz}, 1 \mathrm{H}), 5.32$ $(\mathrm{dd}, \mathrm{J}=15.5,7.9 \mathrm{~Hz}, 1 \mathrm{H}), 5.17(\mathrm{~d}, \mathrm{~J}=2.4 \mathrm{~Hz}, 1 \mathrm{H}), 4.73(\mathrm{~d}, \mathrm{~J}=6.8 \mathrm{~Hz}, 1 \mathrm{H}), 4.68(\mathrm{~d}, \mathrm{~J}=$ $6.8 \mathrm{~Hz}, 1 \mathrm{H}), 4.63(\mathrm{t}, \mathrm{J}=6.6 \mathrm{~Hz}, 1 \mathrm{H}), 3.75(\mathrm{td}, \mathrm{J}=10.2,3.3 \mathrm{~Hz}, 1 \mathrm{H}), 3.62(\mathrm{t}, \mathrm{J}=2.6 \mathrm{~Hz}$, 1H), 3.52 (d, J = 9.6 Hz, 1H), 3.41 (s, 3H), $3.36(\mathrm{~s}, 1 \mathrm{H}), 3.19$ (dd, J = 7.0, 4.6 Hz, 1H), $2.78(\mathrm{~m}, 1 \mathrm{H}), 2.44(\mathrm{~m}, 1 \mathrm{H}), 1.98-1.60(\mathrm{~m}, 5 \mathrm{H}), 1.19(\mathrm{~d}, \mathrm{~J}=7.1 \mathrm{~Hz}, 3 \mathrm{H}), 1.18-1.07(\mathrm{~m}$, 5H), 1.03 (d, J = 6.8 Hz, 3H), 0.93 (d, J = 7.0 Hz, 3H), 0.90 (s, 9H), 0.88 (s, 9H), 0.79 (d, $\mathrm{J}=6.8 \mathrm{~Hz}, 3 \mathrm{H}), 0.09$ (s, 3H), 0.07 (s, 3H), 0.05 (s, 3H), 0.03 (s, 3H). ${ }^{13} \mathrm{C} \mathrm{NMR}(75 \mathrm{MHz}$, $\left.\mathrm{CDCl}_{3}\right) \delta 136.8,130.5,98.3,94.4,86.8,85.8,82.7,76.7,76.3,62.5,56.4,43.1,41.3,40.9$, $40.4,35.1,33.29,33.26,30.1,26.4,26.3,26.1,25.8,18.3,18.21,18.18,16.7,14.0,9.6$, 4.2, -4.3, -4.8, -5.3; IR (neat) $v_{\max } 3570$ (broad), 2945, 2834, 1105, 1049, 1035; HRMS (FAB) calcd for $\mathrm{C}_{38} \mathrm{H}_{71} \mathrm{O}_{6} \mathrm{Si}_{2}\left[(\mathrm{M}-\mathrm{H})^{+}\right]$679.4789, found 679.4774 .

MOM Ether of Alcohol 11. To a solution of alcohol $11(30.0 \mathrm{mg}, 0.0435 \mathrm{mmol})$ in 1 $\mathrm{mL}$ of methylene chloride at $0{ }^{\circ} \mathrm{C}$ was added $\mathrm{MOMCl}(35.0 \mathrm{mg}, 32.4 \mu \mathrm{L}, 0.435 \mathrm{mmol})$, ${ }^{i} \operatorname{Pr}_{2} \mathrm{NEt}(112 \mathrm{mg}, 152 \mu \mathrm{L}, 0.870 \mathrm{mmol})$ and DMAP $(5.3 \mathrm{mg}, 0.0435 \mathrm{mmol})$. The reaction was stirred for $2 \mathrm{~h}$ at $0{ }^{\circ} \mathrm{C}$ and then $16 \mathrm{~h}$ at room temperature. A saturated solution of sodium carbonate $(1 \mathrm{~mL})$ was added to quench the reaction. The aqueous phase was extracted with methylene chloride $(10 \mathrm{~mL} \times 3)$ and the organic phases were combined, dried $\left(\mathrm{MgSO}_{4}\right)$, and concentrated. Chromatography (HE: EA = 10:1) gave MOM ether of $11(28.1 \mathrm{mg}, 87 \%)$ as a colorless oil. ${ }^{1} \mathrm{H}$ NMR $\left(400 \mathrm{MHz}, \mathrm{CDCl}_{3}\right) \delta 5.42(\mathrm{dd}, \mathrm{J}=15.5$, $6.6 \mathrm{~Hz}, 1 \mathrm{H}), 5.28(\mathrm{ddd}, \mathrm{J}=15.5,7.9,1.1 \mathrm{~Hz}, 1 \mathrm{H}), 5.17(\mathrm{~d}, \mathrm{~J}=2.6 \mathrm{~Hz}, 1 \mathrm{H}), 4.92(\mathrm{~d}, \mathrm{~J}=$ $7.0 \mathrm{~Hz}, 1 \mathrm{H}), 4.72(\mathrm{~d}, \mathrm{~J}=6.8 \mathrm{~Hz}, 1 \mathrm{H}), 4.64(\mathrm{~d}, \mathrm{~J}=6.8 \mathrm{~Hz}, 1 \mathrm{H}), 4.60(\mathrm{~d}, \mathrm{~J}=6.8 \mathrm{~Hz}, 1 \mathrm{H})$, 
$4.54(\mathrm{~m}, 1 \mathrm{H}), 3.72(\mathrm{td}, \mathrm{J}=10.0,1.5 \mathrm{~Hz}, 1 \mathrm{H}), 3.64$ (t, J = 2.6 Hz, 1H), 3.40 (s, 3H), 3.38 (s, $3 \mathrm{H}), 3.14(\mathrm{dd}, \mathrm{J}=7.0,4.4 \mathrm{~Hz}, 1 \mathrm{H}), 2.75(\mathrm{~m}, 1 \mathrm{H}), 2.42(\mathrm{~m}, 1 \mathrm{H}), 1.98-1.60(\mathrm{~m}, 5 \mathrm{H}), 1.17$ $(\mathrm{d}, \mathrm{J}=7.0 \mathrm{~Hz}, 3 \mathrm{H}), 1.18-1.07(\mathrm{~m}, 5 \mathrm{H}), 1.01(\mathrm{~d}, \mathrm{~J}=6.7 \mathrm{~Hz}, 3 \mathrm{H}), 0.93(\mathrm{~d}, \mathrm{~J}=7.0 \mathrm{~Hz}, 3 \mathrm{H})$, 0.89 (s, 9H), 0.88 (s, 9H), 0.81 (d, J = 6.8 Hz, 3H), 0.12 (s, 3H), 0.08 (s, 3H), 0.05 (s, 3H), 0.03 (s, 3H). ${ }^{13} \mathrm{C}$ NMR $\left(75 \mathrm{MHz}, \mathrm{CDCl}_{3}\right) \delta 136.8,130.5$, 98.3, 94.6, 94.4, 87.1, 85.9, $81.2,77.1,72.1,63.0,56.3,56.1,43.2,40.9,40.64,40.57,34.5,33.28,33.27,30.2,26.4$, $26.3,26.04,26.01,18.30,18.28,18.24,16.8,14.1,9.7,-3.8,-4.2,-4.7,-5.2$; IR (neat) $v_{\max } 2927,2856,1103,1050,1035$; HRMS (ES+) calcd for $\mathrm{C}_{40} \mathrm{H}_{76} \mathrm{O}_{7} \mathrm{NaSi}_{2}\left[(\mathrm{M}+\mathrm{Na})^{+}\right]$ 747.5027 , found 747.5049 .

Alcohol 18. A solution of Sudan red 7B (40 $\mu \mathrm{L}, 0.05 \%$ in methanol) was added to a solution of the MOM ether of $11(18.0 \mathrm{mg}, 0.0245 \mathrm{mmol})$ in $10 \mathrm{~mL}$ of methylene chloride. The resulted pink solution was cooled to $-78{ }^{\circ} \mathrm{C} . \mathrm{O}_{3}$ was bubbled into the solution until the pink color disappeared. The solution was flushed with argon for $10 \mathrm{~min}$ at $-78{ }^{\circ} \mathrm{C}$ and $\mathrm{NaBH}_{4}(46.3 \mathrm{mg}, 1.23 \mathrm{mmol})$ in $10 \mathrm{~mL}$ of methanol was added. The mixture was stirred for $4 \mathrm{~h}$ at $-78{ }^{\circ} \mathrm{C}$ and then $4 \mathrm{~h}$ at $0{ }^{\circ} \mathrm{C}$. The reaction was then quenched with water $(1 \mathrm{~mL})$. The aqueous phase was extracted with ether and the organic phases were combined, dried $\left(\mathrm{MgSO}_{4}\right)$. Chromatography $(\mathrm{HE}: \mathrm{EA}=2: 1)$ gave alcohol $18(14.2 \mathrm{mg}, 90 \%)$ as a colorless oil. ${ }^{1} \mathrm{H}$ NMR $\left(75 \mathrm{MHz}, \mathrm{CDCl}_{3}\right) \delta 5.18(\mathrm{~d}, \mathrm{~J}=2.6 \mathrm{~Hz}$, 1H), 4.96 (d, J = 7.0 Hz, 1H), 4.89 (d, J = 6.8 Hz, 1H), 4.67 (d, J = 6.8 Hz, 1H), 4.61 (d, J $=6.8 \mathrm{~Hz}, 1 \mathrm{H}), 4.57(\mathrm{~m}, 1 \mathrm{H}), 3.72(\mathrm{td}, \mathrm{J}=10.0,1.5 \mathrm{~Hz}, 1 \mathrm{H}), 3.61(\mathrm{~m}, 1 \mathrm{H}), 3.53(\mathrm{~m}, 1 \mathrm{H})$, $3.43(\mathrm{~s}, 3 \mathrm{H}), 3.38$ (s, 3H), $3.04(\mathrm{dd}, \mathrm{J}=7.0,4.4 \mathrm{~Hz}, 1 \mathrm{H}), 2.75(\mathrm{~m}, 1 \mathrm{H}), 1.95(\mathrm{~m}, 2 \mathrm{H}), 1.78$ (m, 2H), $1.64(\mathrm{~m}, 1 \mathrm{H}), 1.12$ (d, J = 7.0 Hz, 3H), 0.93 (d, J = 7.0 Hz, 3H), 0.89 (s, 9H), $0.88(\mathrm{~s}, 9 \mathrm{H}), 0.80(\mathrm{~m}, 6 \mathrm{H}), 0.11(\mathrm{~s}, 3 \mathrm{H}), 0.08(\mathrm{~s}, 3 \mathrm{H}), 0.05(\mathrm{~s}, 3 \mathrm{H}), 0.03(\mathrm{~s}, 3 \mathrm{H}) .{ }^{13} \mathrm{C} \mathrm{NMR}$ $\left(100 \mathrm{MHz}, \mathrm{CDCl}_{3}\right) \delta$ 99.0, 94.7, 94.4, 87.9, 82.2, 81.1, 76.9, 72.0, 64.8, 63.0, 56.4, 56.1, $43.2,40.6,36.8,34.5,29.9,29.8,26.03,26.01,18.3,18.2,17.7,10.0,9.7,-3.8,-4.2$, -4.7, -5.2; IR (neat) $v_{\max } 3450$ (broad), 2858, 1254, 1099, 1035; HRMS (ES+) calcd for $\mathrm{C}_{33} \mathrm{H}_{66} \mathrm{O}_{8} \mathrm{NaSi}_{2}\left[(\mathrm{M}+\mathrm{Na})^{+}\right]$669.4194, found 669.4188. 
Alcohol 19. To a solution of alcohol $18(12.0 \mathrm{mg}, 0.0185 \mathrm{mmol})$ in $5 \mathrm{~mL}$ of hexanes was added Lindlar catalyst $\left(\mathrm{Pd} / \mathrm{CaCO}_{3}\right.$ poisoned with $\left.\mathrm{Pb}, 5 \% \mathrm{Pd}, 50 \mathrm{mg}\right)$, quinoline (5 $\mu \mathrm{L}) . \mathrm{H}_{2}$ was bubbled through the reaction mixture for $20 \mathrm{~min}$ and the resultant suspension was stirred vigorously for $24 \mathrm{~h}$ under a balloon atmosphere of $\mathrm{H}_{2}$. After filtration through Celite with ether, the solution was concentrated. Chromatography (HE: EA = 2: 1) gave compound 19 (11.2 mg, 93\%) as a colorless oil. ${ }^{1} \mathrm{H}$ NMR (400 MHz, $\left.\mathrm{CDCl}_{3}\right) \delta 5.51$ (dd, $\mathrm{J}=11.2,11.0,1 \mathrm{H}), 5.32(\mathrm{dd}, \mathrm{J}=11.0,9.7 \mathrm{~Hz}, 1 \mathrm{H}), 5.22(\mathrm{~d}, \mathrm{~J}=2.6 \mathrm{~Hz}, 1 \mathrm{H}), 4.66(\mathrm{dd}, \mathrm{J}=$ 6.8, 0.7 Hz, 2H), $4.65(\mathrm{~m}, 1 \mathrm{H}), 4.60$ (d, J = 6.6 Hz, 1H), 4.50 (d, J = 6.8 Hz, 1H), 3.80 (td, $\mathrm{J}=10.0,1.5 \mathrm{~Hz}, 1 \mathrm{H}), 3.65(\mathrm{~m}, 1 \mathrm{H}), 3.56(\mathrm{~m}, 1 \mathrm{H}), 3.41(\mathrm{~s}, 3 \mathrm{H}), 3.31(\mathrm{~s}, 3 \mathrm{H}), 3.27(\mathrm{dd}, \mathrm{J}=$ 6.8, $5.7 \mathrm{~Hz}, 1 \mathrm{H}), 3.10(\mathrm{dd}, \mathrm{J}=6.8,5.7 \mathrm{~Hz}, 1 \mathrm{H}), 2.81(\mathrm{~m}, 1 \mathrm{H}), 1.80(\mathrm{~m}, 3 \mathrm{H}), 1.62(\mathrm{~m}, 1 \mathrm{H})$, $1.44(\mathrm{~m}, 1 \mathrm{H}), 1.01$ (d, J = 7.0 Hz, 3H), 0.92 (m, 6H), 0.90 (s, 9H), 0.89 (s, 9H), 0.81 (d, J $=6.8 \mathrm{~Hz}, 6 \mathrm{H}), 0.14(\mathrm{~s}, 3 \mathrm{H}), 0.11(\mathrm{~s}, 3 \mathrm{H}), 0.05(\mathrm{~s}, 3 \mathrm{H}), 0.04(\mathrm{~s}, 3 \mathrm{H}) .{ }^{13} \mathrm{C} \mathrm{NMR}(100 \mathrm{MHz}$, $\left.\mathrm{CDCl}_{3}\right) \delta 135.1,130.7,99.0,94.3,94.2,84.5,76.9,72.5,68.4,65.2,56.4,55.8,43.2,39.9$, $38.9,35.7,34.7,26.00,25.95,18.83,18.24,14.2,12.5,9.7,-4.0,-4.2,-4.7,-5.2$; IR (neat) $v_{\max } 3450$ (broad), 2858, 1254, 1099, 1035; HRMS (FAB) calcd for $\mathrm{C}_{33} \mathrm{H}_{69} \mathrm{O}_{8} \mathrm{Si}_{2}$ $\left[(\mathrm{M}+\mathrm{H})^{+}\right] 649.4531$, found 649.4514 .

Aldehyde 10. To a stirred mixture of alcohol $19(36.0 \mathrm{mg}, 0.0550 \mathrm{mmol})$ and powdered molecular sieves 4A $(50 \mathrm{mg})$ in $1.0 \mathrm{~mL}$ of methylene chloride were added NMO (40.0 $\mathrm{mg}, 0.340 \mathrm{mmol})$ followed by TPAP $(15.0 \mathrm{mg}, 0.0440 \mathrm{mmol})$. After stirring at $\mathrm{rt}$. for 30 min, the mixture was filtered through a silica gel column (HE: EA =10:1) gave aldehyde $10(32.5 \mathrm{mg}, 90 \%)$ as a colorless oil. ${ }^{1} \mathrm{H} \mathrm{NMR}\left(500 \mathrm{MHz}, \mathrm{CDCl}_{3}\right) \delta 9.78(\mathrm{~s}, 1 \mathrm{H}), 5.51(\mathrm{dd}$, $\mathrm{J}=11.2,10.8,1 \mathrm{H}), 5.36(\mathrm{dd}, \mathrm{J}=10.8,9.2 \mathrm{~Hz}, 1 \mathrm{H}), 5.21(\mathrm{~d}, \mathrm{~J}=2.5 \mathrm{~Hz}, 1 \mathrm{H}), 4.66(\mathrm{dd}, \mathrm{J}=$ 6.8, 0.7 Hz, 2H), $4.65(\mathrm{~m}, 1 \mathrm{H}), 4.60$ (d, J = 6.8 Hz, 1H), 4.53 (d, J = 6.4 Hz, 1H), 3.85 (td, $\mathrm{J}=10.0,1.5 \mathrm{~Hz}, 1 \mathrm{H}), 3.75(\mathrm{t}, \mathrm{J}=10.2 \mathrm{~Hz}, 1 \mathrm{H}), 3.65(\mathrm{t}, \mathrm{J}=2.4 \mathrm{~Hz} 1 \mathrm{H}), 3.38(\mathrm{~s}, 3 \mathrm{H}), 3.32$ $(\mathrm{s}, 3 \mathrm{H}), 3.10(\mathrm{dd}, \mathrm{J}=6.8,5.7 \mathrm{~Hz}, 1 \mathrm{H}), 2.85(\mathrm{~m}, 1 \mathrm{H}), 2.56(\mathrm{~m}, 1 \mathrm{H}), 1.78(\mathrm{~m}, 1 \mathrm{H}), 1.44(\mathrm{~m}$, 1H), $1.14(\mathrm{~d}, \mathrm{~J}=7.3 \mathrm{~Hz}, 3 \mathrm{H}), 1.05$ (d, J = 6.8 Hz, 3H), 0.92 (d, J = 6.8 Hz, 3H) 0.90 (s, 9H), $0.89(\mathrm{~s}, 9 \mathrm{H}), 0.82(\mathrm{~d}, \mathrm{~J}=6.8 \mathrm{~Hz}, 3 \mathrm{H}), 0.13(\mathrm{~s}, 3 \mathrm{H}), 0.10(\mathrm{~s}, 3 \mathrm{H}), 0.06(\mathrm{~s}, 3 \mathrm{H}), 0.05$ (s, $3 \mathrm{H}) .{ }^{13} \mathrm{C}$ NMR $\left(100 \mathrm{MHz}, \mathrm{CDCl}_{3}\right) \delta 204.3,133.4,132.1,97.7,94.5,94.1,81.9,76.9$, 
72.0, 68.8, 56.1, 55.7, 43.2, 39.7, 35.5, 34.8, 26.0, 25.9, 18.9, 18.3, 18.1, 14.2, 9.7, 8.6, $3.8,-4.3,-4.7,-5.3$; IR (neat) $v_{\max } 2930,2858,1731,1254,1099,1035$.

Vinyl Iodide 2. To a suspension of ethyl triphenyphosphonium bromide $(930 \mathrm{mg}, 2.52$ $\mathrm{mmol})$ in THF $(25 \mathrm{~mL})$ was added BuLi (1.00 mL, 2.5 M in hexane, $2.5 \mathrm{mmol})$ dropwise at room temperature. After $10 \mathrm{~min}$, the resultant red solution was transferred to a cold solution $\left(-78^{\circ} \mathrm{C}\right)$ of iodine $(636 \mathrm{mg}, 2.52 \mathrm{mmol})$ in $60 \mathrm{~mL}$ of THF. After stirring $30 \mathrm{~min}$ at $-78{ }^{\circ} \mathrm{C}$, The yellow slurry was warmed slowly to room temperature. Hexane $(60 \mathrm{~mL})$ was added to the flask while stirring. The formed yellow solid was collected by filtration and washed with hexane (30 mL X 2). After drying in vacuo, the $\mathrm{Ph}_{3} \mathrm{P}\left(\mathrm{CHICH}_{3}\right) \mathrm{I}$ was obtained as a yellow solid $(1.17 \mathrm{~g}, 85 \%)$ which could be stored for at least two months under argon.

To a suspension $\mathrm{Ph}_{3} \mathrm{P}\left(\mathrm{CHICH}_{3}\right) \mathrm{I}$ ( $54.5 \mathrm{mg}, 0.10 \mathrm{mmol}$ ) in $2 \mathrm{~mL}$ of THF was added NaHMDS (100 $\mu \mathrm{L}, 1.0 \mathrm{M}$ in THF) at $-23{ }^{\circ} \mathrm{C}$, the resultant red solution was stirred for 30 min at $-23{ }^{\circ} \mathrm{C}$ and then cooled to $-30{ }^{\circ} \mathrm{C}$. A solution of aldehyde $10(20.0 \mathrm{mg}, 0.0309$ $\mathrm{mmol})$ in THF $(1 \mathrm{~mL})$ was added via cannula. The reaction mixture was stirred for $2 \mathrm{~h}$ at $-30{ }^{\circ} \mathrm{C}$ and then warmed to $4^{\circ} \mathrm{C}$ over $1 \mathrm{~h}$. $\mathrm{MeOH}(0.2 \mathrm{~mL})$ was added to quench the reaction. The insoluable material was removed by filtration through a short silica plug. Concentration followed by chromatography (HE: EA = 20: 1) gave compound $2(9.7 \mathrm{mg}$, $40 \%, 9: 1 \mathrm{Z} / \mathrm{E})$ as a colorless oil. ${ }^{1} \mathrm{H} \mathrm{NMR}\left(500 \mathrm{MHz}, \mathrm{CDCl}_{3}\right) \delta 5.58(\mathrm{dd}, \mathrm{J}=11.2,10.8$, 1H), $5.28(\mathrm{~m}, 2 \mathrm{H}), 5.18(\mathrm{~d}, \mathrm{~J}=2.5 \mathrm{~Hz}, 1 \mathrm{H}), 4.64(\mathrm{~m}, 4 \mathrm{H}), 4.51(\mathrm{~d}, \mathrm{~J}=6.8 \mathrm{~Hz}, 1 \mathrm{H}), 3.78$ (td, J = 10.0, 1.5 Hz, 1H), $3.63(\mathrm{t}, \mathrm{J}=2.4 \mathrm{~Hz} 1 \mathrm{H}), 3.39$ (s, 3H), 3.38 (s, 3H), 3.30 (dd, J = 6.8, $5.7 \mathrm{~Hz}, 1 \mathrm{H}), 2.72(\mathrm{~m}, 1 \mathrm{H}), 2.49(\mathrm{~s}, 3 \mathrm{H}), 1.78(\mathrm{~m}, 1 \mathrm{H}), 1.44(\mathrm{~m}, 1 \mathrm{H}), 1.04(\mathrm{~d}, \mathrm{~J}=6.9$ $\mathrm{Hz}, 3 \mathrm{H}), 1.00$ (d, J = 6.8 Hz, 3H), 0.91 (d, J = 7.2 Hz, 3H) 0.90 (s, 9H), 0.88 (s, 9H), 0.81 $(\mathrm{d}, \mathrm{J}=6.8 \mathrm{~Hz}, 3 \mathrm{H}), 0.12$ (s, 3H), 0.09 (s, 3H), 0.04 (s, 3H), 0.02 (s, 3H). ${ }^{13} \mathrm{C}$ NMR $(100$ $\left.\mathrm{MHz}, \mathrm{CDCl}_{3}\right) \delta 138.3,134.2,131.1,100.5,98.2,94.3,94.2,85.9,76.9,71.9,69.0,56.4$, $55.8,44.7,43.2,39.9,35.4,34.6,34.0,26.0,18.8,18.3,15.3,14.3,9.8,-3.7,-4.3,-4.7$, 5.3; IR (neat) $v_{\max }$ 2930, 2857, 1151, 1097, 1038; HRMS (ES+) calcd for $\mathrm{C}_{35} \mathrm{H}_{69} \mathrm{O}_{7} \mathrm{NaSi}_{2} \mathrm{I}\left[(\mathrm{M}+\mathrm{Na})^{+}\right]$807.3524, found 807.3522. 
Assignment of stereochemistry to alcohol 15 and its epimer by application of the Rychnovsky method ${ }^{1}$ to the corresponding diols

The major isomer of the product of the brown allylboration of aldehyde $\mathbf{1 4}$ was deprotected and the resulting diol $\mathbf{2 0}$ was derivatized as the acetonide 22, a compound with ${ }^{13} \mathrm{C}$ signals at 30.3 and $19.8 \mathrm{ppm}$ for the gem methyl carbons and $97.9 \mathrm{ppm}$ for the ketal carbon. The minor isomer was subjected to the same procedure, affording an acetonide 23 with ${ }^{13} \mathrm{C}$ signals at 25.5 and $23.7 \mathrm{ppm}$ for the gem methyl carbons and $100.2 \mathrm{ppm}$ for the ketal carbon. These results are consistent with the assignment of structure of the major stereoisomer from the allylation as the syn, anti, anti alcohol 15 and the minor isomer as its (4R)-epimer.
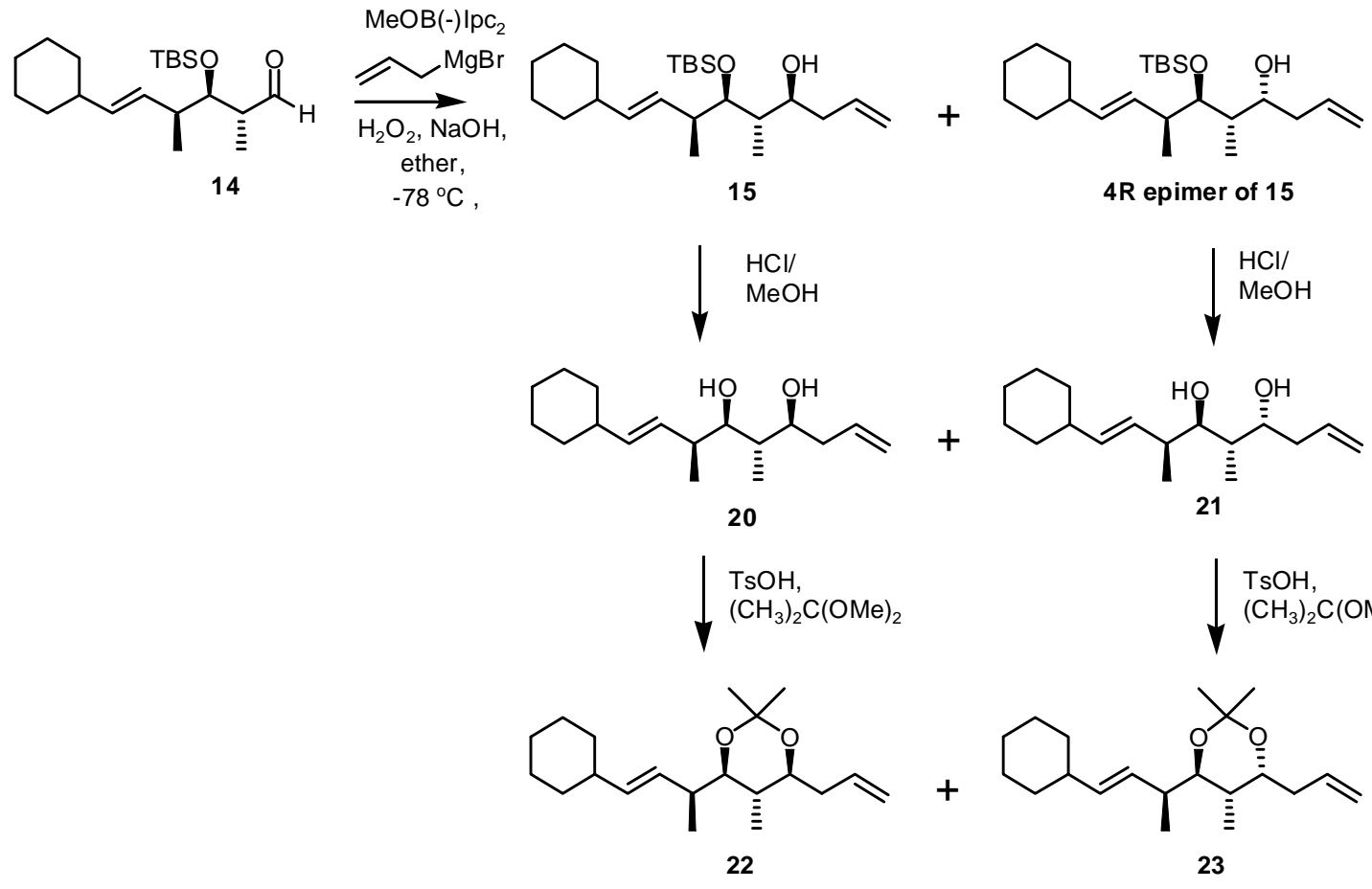

21
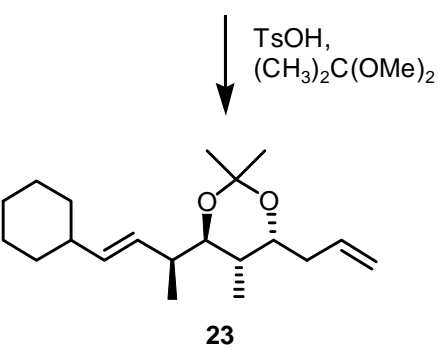

\section{Experimental Section:}

Diol 20. To a solution of alcohol $15(15.0 \mathrm{mg}, 0.0394 \mathrm{mmol})$ in $\mathrm{MeOH} / \mathrm{ether}(1 \mathrm{~mL} / 1$ $\mathrm{mL}$ ) was added $4 \mathrm{~N} \mathrm{HCl}$ aqueous solution $(2 \mathrm{~mL})$ at room temperature. The reaction mixture was stirred for $24 \mathrm{~h}$ at room temperature and then poured into ether and saturated

\footnotetext{
1 (a) Rychnovsky, S. D.; Rogers, B.; Yang, G. J. Org. Chem. 1993, 58, 3511. (b) Rychnovsky, S. D.; Skalitzky, D. J. Tetrahedron Lett. 1990, 31, 945. (c) Evans, D. A.; Rieger, D. L.; Gage, J. R. Tetrahedron Lett. 1990, 31, 7099.
} 
sodium bicarbonate solution $(10 \mathrm{~mL} / 10 \mathrm{~mL})$. The organic phase was separated and dried $\left(\mathrm{Na}_{2} \mathrm{SO}_{4}\right)$. Chromatography $(\mathrm{HE}: \mathrm{EA}=2: 1)$ gave compound $20(9.0 \mathrm{mg}, 86 \%)$ as a colorless oil. ${ }^{1} \mathrm{H}$ NMR $\left(300 \mathrm{MHz}, \mathrm{CDCl}_{3}\right) \delta 5.90(\mathrm{~m}, 1 \mathrm{H}), 5.44(\mathrm{~m}, 2 \mathrm{H}), 5.17(\mathrm{~d}, \mathrm{~J}=1.5$ $\mathrm{Hz}, 1 \mathrm{H}), 5.11(\mathrm{~m}, 1 \mathrm{H}), 3.77$ (tt, J = 8.5, $2.7 \mathrm{~Hz}, 1 \mathrm{H}), 3.50$ (dd, J = 6.0, 4.6 Hz, 1H), 3.48 (broad, 1H), 2.58 (broad, 1H), 2.52-2.38 (m, 2H), 2.14-2.08 (m, 1H), 2.02-1.95 (m, 1H), 1.80-1.60 (m, 6H), 1.38-1.01, (m, 5H), 0.97 (d, J = 6.9 Hz, 3H), 0.80 (d, J = 6.8 Hz, 3H). ${ }^{13} \mathrm{C}$ NMR $\left(75 \mathrm{MHz}, \mathrm{CDCl}_{3}\right) \delta 137.8,135.1,130.4,117.7,79.0,75.1,40.8,40.2,39.1$, $38.4,33.3,33.2,26.1,26.0,12.7,11.1$; HRMS (FAB) calcd for $\mathrm{C}_{17} \mathrm{H}_{31} \mathrm{O}_{2}\left[(\mathrm{M}+\mathrm{H})^{+}\right]$ 267.2324, found 267.2305.

Diol 21. Compound 21 was synthesized in a similar procedure using the (4R)-epimer of alcohol 15 as starting material. ${ }^{1} \mathrm{H}$ NMR $\left(300 \mathrm{MHz}, \mathrm{CDCl}_{3}\right) \delta 5.80(\mathrm{~m}, 1 \mathrm{H}), 5.42(\mathrm{dd}, \mathrm{J}=$ 15.4, $6.0 \mathrm{~Hz}, 1 \mathrm{H}), 5.25$ (ddd, J = 15.4, 8.0, 1.1 Hz), 5.10 (m, 2H), $4.02(\mathrm{~m}, 1 \mathrm{H}), 3.40$ (m, $1 \mathrm{H}), 3.45(\mathrm{~s}, 1 \mathrm{H}), 2.58(\mathrm{~m}, 1 \mathrm{H}), 2.42(\mathrm{~m}, 1 \mathrm{H}), 2.40-2.10(\mathrm{~m}, 3 \mathrm{H}), 1.98-1.60(\mathrm{~m}, 7 \mathrm{H})$, 1.38-1.01, (m, 5H), $1.01(\mathrm{~d}, \mathrm{~J}=6.9 \mathrm{~Hz}, 3 \mathrm{H}), 0.98(\mathrm{~d}, \mathrm{~J}=6.9 \mathrm{~Hz}, 3 \mathrm{H}) ;{ }^{13} \mathrm{C}$ NMR $(75$ $\left.\mathrm{MHz}, \mathrm{CDCl}_{3}\right) \delta 137.6,135.8,130.4,117.8,89.5,71.7,41.1,40.6,39.3,38.0,33.5,33.4$, 26.4, 26.3, 15.7, 11.5; HRMS (FAB) calcd for $\mathrm{C}_{17} \mathrm{H}_{31} \mathrm{O}_{2}\left[(\mathrm{M}+\mathrm{H})^{+}\right] 267.2324$, found 267.2311 .

Acetonide 22. To a dry round bottom flask was added diol $20(6.0 \mathrm{mg}, 0.0225 \mathrm{mmol})$, TsOH (3mg, $0.0168 \mathrm{mmol})$ and $\left(\mathrm{CH}_{3}\right)_{2} \mathrm{C}\left(\mathrm{OCH}_{3}\right)_{2}(1 \mathrm{~mL})$. The reaction mixture was tirred for $1 \mathrm{~h}$ at room temperature and then poured into ether/saturated sodium bicarbonate aqueous solution $(5 \mathrm{~mL} / 5 \mathrm{~mL})$. The organic phase was separated, dried $\left(\mathrm{MgSO}_{4}\right)$ and concentrated. Chromatography (HE: EA = 10: 1) gave compound $22(5.2 \mathrm{mg}, 76 \%)$ as a colorless oil. ${ }^{1} \mathrm{H}$ NMR $\left(400 \mathrm{MHz}, \mathrm{CDCl}_{3}\right) \delta 5.91(\mathrm{~m}, 1 \mathrm{H}), 5.46(\mathrm{dd}, \mathrm{J}=15.6,7.8 \mathrm{~Hz}, 1 \mathrm{H})$, $5.33(\mathrm{dd}, \mathrm{J}=15.6,6.4 \mathrm{~Hz}, 1 \mathrm{H}), 5.04(\mathrm{~m}, 2 \mathrm{H}), 3.51(\mathrm{~m}, 1 \mathrm{H}), 3.40(\mathrm{dd}, \mathrm{J}=10.2,2.5 \mathrm{~Hz}$, $1 \mathrm{H}), 2.42-2.22(\mathrm{~m}, 2 \mathrm{H}), 2.20-2.12(\mathrm{~m}, 1 \mathrm{H}), 1.98-1.85(\mathrm{~m}, 1 \mathrm{H}), 1.75-1.60(\mathrm{~m}, 6 \mathrm{H}), 1.37(\mathrm{~S}$, $3 \mathrm{H}), 1.35(\mathrm{~S}, 3 \mathrm{H}), 1.30-1.01,(\mathrm{~m}, 5 \mathrm{H}), 0.92(\mathrm{~d}, \mathrm{~J}=7.2 \mathrm{~Hz}, 3 \mathrm{H}), 0.76(\mathrm{~d}, \mathrm{~J}=6.4 \mathrm{~Hz}, 3 \mathrm{H})$. ${ }^{13} \mathrm{C}$ NMR $\left(100 \mathrm{MHz}, \mathrm{CDCl}_{3}\right) \delta 135.5,135.4,132.1,116.3,97.9,77.7,74.4,40.8,37.8$, $35.3,33.5,30.3,26.5,26.3,19.8,13.7,12.1$; HRMS (FAB) calcd for $\mathrm{C}_{17} \mathrm{H}_{31} \mathrm{O}_{2}\left[(\mathrm{M}-\mathrm{H})^{+}\right]$ 305.2481 , found 305.2474 
Acetonide 23 was synthesized by a procedure similar to that above using diol $\mathbf{2 1}$ as starting material. ${ }^{1} \mathrm{H}$ NMR $\left(300 \mathrm{MHz}, \mathrm{CDCl}_{3}\right) \delta 5.80(\mathrm{~m}, 1 \mathrm{H}), 5.42(\mathrm{dd}, \mathrm{J}=15.4,6.0 \mathrm{~Hz}$, $1 \mathrm{H}), 5.28(\mathrm{ddd}, \mathrm{J}=15.4,8.0,1.1 \mathrm{~Hz}), 5.05(\mathrm{~m}, 2 \mathrm{H}), 3.80(\mathrm{~m}, 1 \mathrm{H}), 3.05(\mathrm{~m}, 1 \mathrm{H}), 2.25-$ $2.00(\mathrm{~m}, 4 \mathrm{H}), 2.00-1.82(\mathrm{~m}, 1 \mathrm{H}), 1.80-1.60(\mathrm{~m}, 7 \mathrm{H}), 1.37(\mathrm{~s}, 6 \mathrm{H}), 1.36-1.01,(\mathrm{~m}, 5 \mathrm{H})$, $1.01(\mathrm{~d}, \mathrm{~J}=6.9 \mathrm{~Hz}, 3 \mathrm{H}), 0.82(\mathrm{~d}, \mathrm{~J}=6.9 \mathrm{~Hz}, 3 \mathrm{H}) ;{ }^{13} \mathrm{C} \mathrm{NMR}\left(75 \mathrm{MHz}, \mathrm{CDCl}_{3}\right) \delta 136.6$, 135.4, 129.6, 116.3, 100.2, 78.6, 69.1, 41.7, 40.7, 36.7, 35.2, 33.2, 33.1, 26.2, 26.1, 25.5, 23.7, 16.7, 12.5; HRMS (FAB) calcd for $\mathrm{C}_{17} \mathrm{H}_{31} \mathrm{O}_{2}\left[(\mathrm{M}-\mathrm{H})^{+}\right]$305.2481, found 305.2477.

\section{Assignment of stereochemistry to alcohol 11 and its C-7 epimer by application of the "broadened" version of the advanced Mosher ester method.}

The "advanced Mosher ester method" for determination of absolute stereochemistry at secondary alcohol centers ${ }^{2}$ is regarded as highly reliable. It assigns each of the carbon substituents to a quadrant of space on the basis of a comparison of the ${ }^{1} \mathrm{H} \mathrm{nmr} \mathrm{spectra}$ of the (S)- and (R)-Mosher esters. Thus, in the (S)-Mosher ester, "Model A," the protons $\mathrm{H}_{\mathrm{A}}, \mathrm{H}_{\mathrm{B}}$, and $\mathrm{H}_{\mathrm{C}}$ which are connected to the carbon in the quadrant on the right will have higher chemical shifts than $\mathrm{H}_{\mathrm{A}}, \mathrm{H}_{\mathrm{B}}$, and $\mathrm{H}_{\mathrm{C}}$ in the corresponding (S)-Mosher ester, "Model B". Also, $\mathrm{H}_{\mathrm{X}}, \mathrm{H}_{\mathrm{Y}}$, and $\mathrm{H}_{\mathrm{Z}}$, the protons connected to the carbon in the quadrant on the left in "Model A," will have lower chemical shifts than those in the corresponding (R)-Mosher ester "Model B." In the Figure, $\Delta \delta_{\mathrm{H}}=\delta_{\mathrm{S}}-\delta_{\mathrm{R}}$.

2 (a) Ohtani, I.; Kusumi, T.; Kashman, Y.; Kakisawa, H. J. Am. Chem. Soc. 1991,113, 4092. (b) Dale, J. A.; Mosher, H. S. J. Am. Chem. Soc. 1973, 95, 512. 


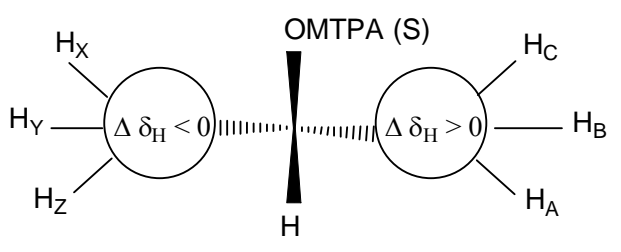

Model A

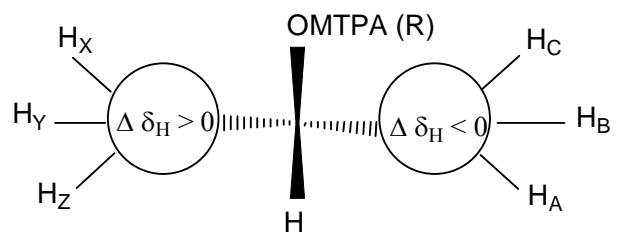

Model B

Figure derived from references 2 (a) and (b).

In the "modified advanced Mosher ester method," epimeric alcohols are subjected to esterification with either the (R)- or the (S)-Mosher acid chloride. This affords a pair of diastereomers that may be portrayed as "Model B" and "Model B'."
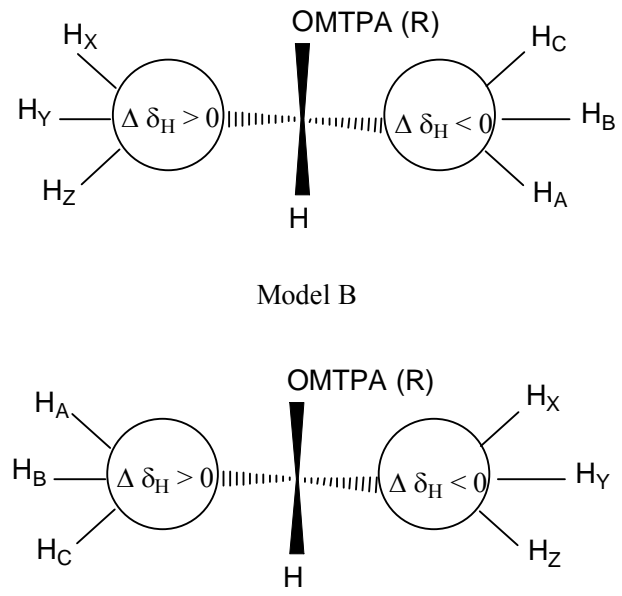

Model B'

As noted by McLaughlin et al. ${ }^{3}$, the "advanced Mosher ester method" and the "modified advanced Mosher ester method" are not equivalent but they are based on the same principle.

${ }^{3}$ Shi, G.; He, K.; Liu, X.;.Ye, Q.; MacDougal, J. M.; McLaughlin, J. L. Natural Product Lett., 1997, 10, 125. 
Each epimeric alcohol from the alkynylation of aldehyde $\mathbf{1 2}$ was converted to the corresponding (R)-Mosher ester by treatment with (S)- $\alpha$-methoxy- $\alpha$ trifluoromethyl)phenyl acetyl chloride (see experimental protocol below). In the (R)Mosher ester of the major isomer (11), the signal for the C-10 methyl group appeared at $1.137 \mathrm{ppm}$ and the signal for the C-4 methyl group appeared at $0.786 \mathrm{ppm}$; in the (R)Mosher ester of the minor isomer (the 7R-epimer of 11), the chemical shift of the C-10 methyl group appeared at $1.186 \mathrm{ppm}$ and the signal for the C-4 methyl group appeared at 0.744 ppm.

These data are consistent with an (S)-configuration in the major product and an (R) configuration in the minor product. Both the R-Mosher ester of the 7S alcohol (11) and the R-Mosher ester of the 7R alcohol are shown in the conformation used for the assignment of absolute stereochemistry. The diagnostic chemical shifts are shown on the structures.

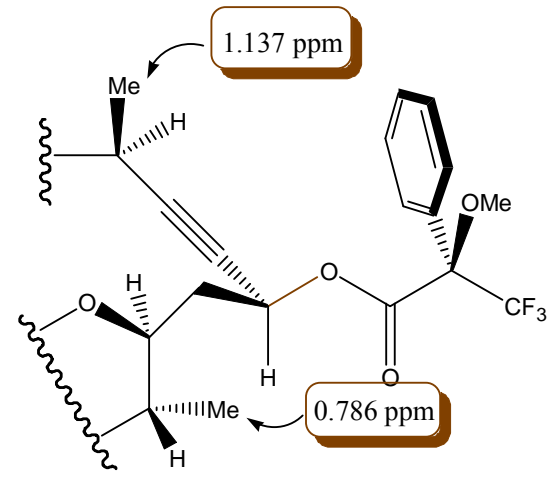

R-Mosher Ester of 7S Alcohol

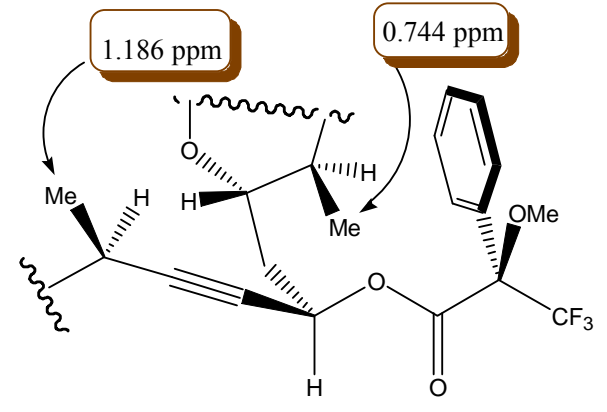

R-Mosher Ester of 7R Alcohol

\section{Experimental Section}

(R)-Mosher Ester of the Alcohol 11. The major product of the addition of alkyne 13 to aldehyde 12 (13.6 mg, $0.02 \mathrm{mmol})$ and DMAP (11.0 mg, $0.12 \mathrm{mmol})$ was dissolved in $\mathrm{CH}_{2} \mathrm{Cl}_{2}(1 \mathrm{~mL})$ and $S$ - $\alpha$-methoxy- $\alpha$-trifluoromethyl phenyl acetyl chloride $(7.5 \mu \mathrm{L}, 0.04$ mmol) was added dropwise. After $2 \mathrm{~h}$ at room temperature, water $(5 \mathrm{~mL})$ and EtOAc $(15$ $\mathrm{mL})$ were added. The organic phase was washed with saturated $\mathrm{NaHCO}_{3}(2 \times 5 \mathrm{~mL})$. The organic phase was isolated, dried $\left(\mathrm{MgSO}_{4}\right)$, and concentrated. The residue was dissolved and washed with solvent $(\mathrm{HE}: \mathrm{EA}=10: 1)$ through a silica gel column to give crude $(\mathrm{R})$ - 
Mosher ester as a colorless oil (14.6 mg, 80\%) for ${ }^{1} \mathrm{H}$ NMR analysis. ${ }^{1} \mathrm{H}$ NMR (300 $\mathrm{MHz}, \mathrm{CDCl}_{3}$ ) C4-Me $\delta 0.786(\mathrm{~d}, \mathrm{~J}=6.9 \mathrm{~Hz}, 3 \mathrm{H}), \mathrm{C} 10-\mathrm{Me} 1.137(\mathrm{~d}, \mathrm{~J}=6.9 \mathrm{~Hz}, 3 \mathrm{H})$.

(R)-Mosher Ester of the 7R-epimer of alcohol 11. The minor product of the addition of alkyne 13 to aldehyde 12 (13.6 mg, $0.02 \mathrm{mmol})$ and DMAP (11.0 mg, $0.12 \mathrm{mmol}$ ) was dissolved in $\mathrm{CH}_{2} \mathrm{Cl}_{2}(1 \mathrm{~mL})$ and $S$ - $\alpha$-methoxy- $\alpha$-trifluoromethyl phenyl acetyl chloride $(7.5 \mu \mathrm{L}, 0.04 \mathrm{mmol})$ was added dropwise. After $2 \mathrm{~h}$ at room temperature, water $(5 \mathrm{~mL})$ and EtOAc $(15 \mathrm{~mL})$ were added. The organic phase was washed with saturated $\mathrm{NaHCO}_{3}$ $(2 \times 5 \mathrm{~mL})$. The organic phase was isolated, dried $\left(\mathrm{MgSO}_{4}\right)$, and concentrated. The residue was dissolved and washed with solvent $(\mathrm{HE}: \mathrm{EA}=10: 1)$ through a silica gel column to give crude Mosher ester as a colorless oil (15.2 mg, 83\%) for ${ }^{1} \mathrm{H}$ NMR analysis. ${ }^{1} \mathrm{H}$ NMR $\left(300 \mathrm{MHz}, \mathrm{CDCl}_{3}\right) \mathrm{C} 4-\mathrm{Me} \delta 0.744(\mathrm{~d}, \mathrm{~J}=6.9 \mathrm{~Hz}, 3 \mathrm{H}), \mathrm{C} 10-\mathrm{Me} 1.186$ $(\mathrm{d}, \mathrm{J}=6.9 \mathrm{~Hz}, 3 \mathrm{H})$. 